\title{
Denoising physiological data collected during multi-band, multi-echo EPI sequences
}

\author{
Katherine L. Bottenhorn'1,2, Taylor Salo 1,2, Michael C. Riedel2,3, Matthew \\ T. Sutherland ${ }^{1,2}$, Jennifer L. Robinson ${ }^{4}$, Erica D. Musser ${ }^{1,2}$, Angela R. \\ Laird ${ }^{2,3}$
}

\section{Abstract}

Collecting physiological data during fMRI experiments can improve fMRI data cleaning and contribute to our understanding of psychophysiological processes; however, these recordings are frequently fraught with artifacts from the MRI pulse sequence. Here, we look at manufacturer recommendations for filtering such artifacts from physiological data collected during single-band, single-echo fMRI sequences and extend these recommendations to address artifacts associated with multiband, multi-echo fMRI sequences. While the magnitude and frequencies of artifacts differ with these aspects of pulse sequences, their effects can be mitigated via application of digital filters focused on the slice collection and repetition time.

Keywords physiology; signal processing; fMRI; psychophysiology; data preprocessing

\footnotetext{
${ }^{1}$ Department of Psychology, Florida International University, Miami, FL, USA

${ }^{2}$ Center for Imaging Science Florida International University, Miami, FL, USA

${ }^{3}$ Department of Physics, Florida International University, Miami, FL, USA

${ }^{4}$ Department of Psychology, Auburn University, Auburn, AL, USA
}

\section{Corresponding Author:}

Angela R. Laird, Department of Physics, Florida International University, AHC4 310, Modesto Maidique Campus, 11200 SW 8th Street, Miami, FL 33199. Phone: 305.348.6737, Fax: 305.348.6700, Email: alaird@fiu.edu 


\section{Introduction}

Physiological recordings collected simultaneously during functional magnetic resonance imaging (fMRI) can add valuable information about a participant's physical state and provide quantitative assessment of psychological phenomena. Furthermore, they offer the opportunity to study relations between the central and autonomic nervous systems (CNS and ANS, respectively) that underlie cognition and behavior. For example, physiological arousal has been assessed during fMRI using heart rate, via electrocardiogram (ECG) recordings and skin conductance, via electrodermal activity (EDA) recordings. Inclusion of such measures may enhance interpretation of studies examining decision making (reviewed in (Wong et al., 2011), typical and disordered affective processing (Goldstein et al., 2005; Kraus et al., 2007; Mulcahy et al., 2019; Shokri-Kojori et al., 2018), pain (Mobascher et al., 2009, 2010; Perlaki et al., 2015), autonomic regulation (Kuoppa et al., 2012; Lane et al., 2013; Napadow et al., 2008; Sclocco et al., 2019; Valenza et al., 2014), and fMRI denoising (Abreu et al., 2017; Hu et al., 2019).

Collecting electrophysiological recordings in the MR environment adds MR-induced artifacts to the recordings. Often, the magnitude of these MR artifacts is much larger than that of the phenomena of interest, necessitating additional data cleaning steps before such data can be used to assess psychophysiological phenomena. Single-echo MRI sequences (Figure 1A) that measure the blood-oxygenation level dependent (BOLD) signal are the norm in fMRI research. However, recent advances in MR technology and denoising approaches are prompting researchers to increasingly turn to multi-echo sequences (Figure 1B), which offer better differentiation between BOLD signal and non-neural noise for improved estimates of brain activation (Boyacioğlu et al., 2015; Kundu et al., 2017; Olafsson et al., 2015). While these sequences arguably offer better quality fMRI data, they require more complex radio frequency (RF) and gradient pulses that introduce added artifacts to simultaneously collected electrophysiological recordings. Furthermore, adding echoes to an MRI sequence introduces further limitations on the temporal resolution of the sequence, requiring a longer repetition time (TR). Using multiband or simultaneous multi-slice (SMS) excitation allows researchers to reduce the amount of time required to acquire a single volume, by acquiring several slices simultaneously, and effectively minimizing the multi-echo temporal constraints on TR. This is an important consideration for human fMRI studies, in which a study's power to detect an effect is linearly related to the number of timepoints in a scan (Desmond \& Glover, 2002). 
A. Single-band, single-echo GRE-EPI

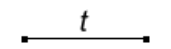

RF

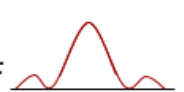

SB \#1

$(\Delta \mathrm{f}=0 \mathrm{~Hz})$

$\mathrm{BW}_{\mathrm{eff}}=\lambda$

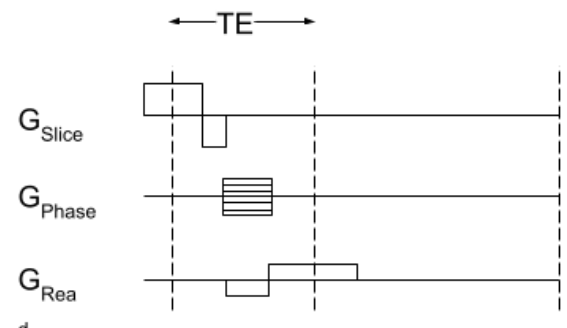

B. Multi-band, multi-echo GRE-EPI

RF
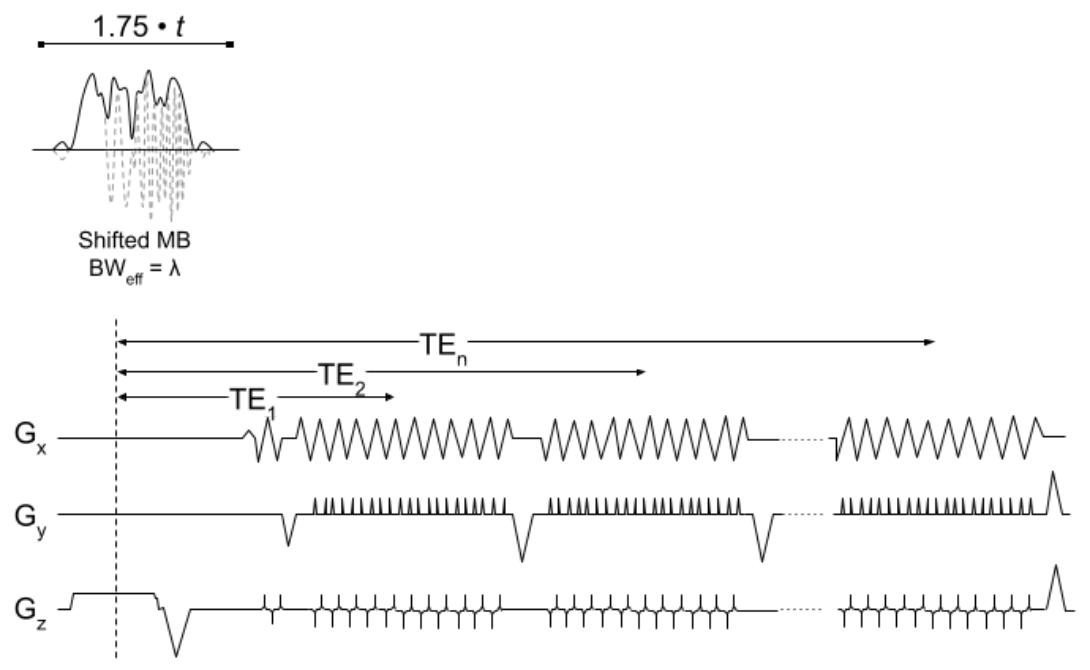

Figure 1. Schematic representation of (A) a single-band, single-echo GRE-EPI pulse sequence and (B) a multi-band, multi-echo GRE-EPI pulse sequence. RF pulse differences (Auerbach et al., 2013) are highlighted in the first row, while differences in time-varying gradient fields (Cohen et al., 2017) are shown in the following three rows.

The present study assessed MR-artifact removal strategies from electrophysiological (i.e., ECG and EDA) data collected during single- and multi-echo multiband EPI scans. Currently, there are few commercial manufacturers of MR-compatible equipment for collecting and filtering electrophysiological data during MRI scans for research purposes. Of these, BIOPAC Systems, Inc. (biopac.com) is a popular choice among neuroimaging researchers and among the only manufacturers to offer recommendations for filtering MR-noise out of concurrently collected electrophysiological data. Our goals were to (1) compare MR-related noise from single- and multi-echo EPI sequences, (2) assess current filtering recommendations in multiband and multi-echo contexts, and (3) if current filtering recommendations appeared insufficient for removing MR-related artifacts from concurrent electrophysiological data, to redefine these recommendations accordingly. To achieve this, we used ECG and EDA data collected during both single- and multi-echo multiband BOLD EPI sequences, from 5 participants across 700+ minutes of scanning. First, data were Fourier transformed to identify MR-artifact frequencies, then digital filters were applied, and cleaned data were compared to data collected in the absence of MR sequences, both visually and quantitatively. We anticipated that MR-artifacts would be greatest during multiband, multi-echo EPI sequences and while current filtering recommendations would mitigate MR-artifacts, adaptations may be necessary for multiband and multi-echo pulse sequences. We expected that adaptations to the slice collection frequency would be necessary, to account for slices collected in parallel. Finally, we make these findings openly available both as interactive online code and easy-to-use command-line software, sharing updated recommendations for removing MR-artifacts from these physiological data in multiband and multi-echo contexts. 


\section{Methods}

\section{Physiological recordings}

Physiological data were collected using MRI-compatible modules, leads, and electrodes from BIOPAC Systems. Data were acquired using a BIOPAC MP150 system, connected to subject leads by two standard MEC-MRI cables that passed through the MRI patch panel via MRI-RFIF filters and ran without loops to the bore, then parallel with the subject. Electrocardiogram (ECG) recordings were collected using radiotranslucent EL508 electrodes with GEL100 and LEAD108 leads, with an ECG100C-MRI amplifier. Electrodes were placed in a 3-lead bipolar monitoring configuration, 6 to 8 inches apart diagonally across the heart from right clavicle to left rib cage, with the ground placed 6 to 8 inches away on the right rib cage. Electrodermal activity (EDA) recordings were collected using radiotranslucent EL509 electrodes with GEL101 and LEAD108 leads, with an EDA100C-MRI amplifier. Leads were placed on the palm of the participant's non-dominant hand, on the thenar and hypothenar eminences. All physiological data were collected at a rate of $2000 \mathrm{~Hz}$, with ECG and EDA collected concurrently from all participants. Physiological data collection began once participants were loaded on the scanner bed and continued until the scanner bed exited the bore after the scanning session, including several minutes per participant of data collected in the absence of an MR pulse sequence.

\section{BOLD EPI Sequences}

Physiological recordings were acquired in the bore of a whole-body 3-Tesla Siemens MAGNETOM Prisma with a 32-channel head/neck coil, during both a multiband, single-echo (MBSE) blood-oxygenation-level-dependent (BOLD) echo planar imaging (EPI) sequence and a multiband, multi-echo (MBME) BOLD EPI sequence.

Multiband, single-echo BOLD EPI sequence. The MBSE sequence used here is the one used by the Adolescent Brain Cognitive Development (ABCD) ${ }^{\mathrm{SM}}$ Study (Casey et al., 2018). In brief, this sequence acquired 60 transverse slices, with an anterior to posterior phase encoding direction, using a single echo $(\mathrm{TE}=30 \mathrm{~ms}$ ) with $\mathrm{TR}=800 \mathrm{~ms}$, a multiband acceleration factor of 6 , interleaved acquisition, in-plane GRAPPA acceleration, and a $52^{\circ}$ flip angle. More information about the scan protocols is available with the curated ABCD data via the NIMH Data Archive (NDA; https://abcdstudy.org/scientists/protocols/). Participants in the current study ( $\mathrm{n}=5$, all female, aged 26-39) completed four 6-minute runs of an emotion regulation task (Blair et al., 2012; Ochsner et al., 2002,2004 ) and two 5-minute runs of rest.

Multiband, multi-echo BOLD EPI sequence. The MBME BOLD EPI sequence used here is from the distribution of multi-band accelerated EPI sequences (Moeller et al., 2010) developed by the Center for Magnetic Resonance Research at the University of Minnesota. The MBME GRE-EPI sequence acquired 48 slices at a $30^{\circ}$ transverse-to-coronal orientation with anterior-to-posterior phase encoding direction at each of 4 echoes $\left(\mathrm{TE}_{1}=11.80 \mathrm{~ms}, \mathrm{TE}_{2}=28.04 \mathrm{~ms}, \mathrm{TE}_{3}=44.28 \mathrm{~ms}, \mathrm{TE}_{4}=60.52 \mathrm{~ms}\right)$ with $\mathrm{TR}=1500 \mathrm{~ms}, \mathrm{a}$ multiband acceleration factor of 3, interleaved acquisition, in-plane GRAPPA acceleration, a $77^{\circ}$ flip

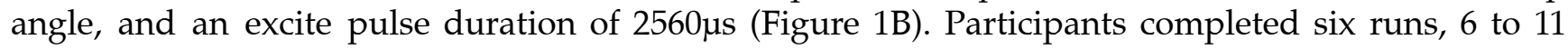
minutes each, of film watching (Duffer \& Duffer, 2017; NBCLab/Film-Viewing-Task, 2019/2020), two runs of the same emotion regulation task (NBCLab/Eirt-Task, 2020/2020), two runs of a probabilistic 
selection task, one 6 minutes and the other 9 minutes (Frank et al., 2004), and two runs of 5 minutes of rest.

The full parameters and fMRI data are available on OpenNeuro.org (https:/ / openneuro.org/datasets/ds002278/versions/1.0.1).

\section{Software tools}

All code used to create and apply these filters is available on GitHub (https://github.com/62442katieb/mbme-physio-denoising), and was written and run using Python 3.7.3. The bioread library (https://github.com/uwmadison-chm/bioread, v. 1.0.4) was used to read in physiological recordings stored in AcqKnowledge format, data were manipulated using Pandas (https://pandas.pydata.org/, v. 1.0.3), digital filters were created and applied using SciPy (https://www.scipy.org, v. 1.4.1; (Virtanen et al., 2020)), and fast Fourier transforms implemented in NumPy (https://numpy.org/, v. 1.18.2; (Harris et al., 2020)).

\section{Denoising electrocardiogram recordings}

Fourier transform was applied to ECG and EDA data collected both in the presence and absence of MR pulse sequences to identify the frequencies of MR-related artifacts. Then, in parallel processing streams for each the ECG and EDA data, we applied digital filters to mitigate the effects of these artifacts on the recordings. First, we applied the manufacturer (i.e., BIOPAC) recommendation for single-band, single-echo sequences: comb band-stop filters at the slice collection frequency and its harmonics up to the Nyquist frequency, and then Fourier transformed the results to assess how these filters mitigated artifacts. This slice collection frequency is defined as:

$$
v_{\text {slice collection }}=\text { number of slices } \div T R
$$

And here, comb band-stop filters were implemented as a series of infinite impulse response (IIR) notch filters to account for the fundamental frequency and its harmonics.

Then, we adjusted the frequencies of these filters to account for additional MR-related artifacts identified in frequency spectra, applied the adjusted filters, and Fourier transformed the results.

Finally, physiological data were compared across steps and to data collected in the absence of MR pulse sequences, using magnitude squared coherence to assess linear dependence across the frequency band in which physiologically-relevant signals were found: $0.5-50 \mathrm{~Hz}$ for ECG and $<0.5 \mathrm{~Hz}$ for EDA.

\section{Results}

\section{Frequencies of MR-related artifacts}

We applied Fourier transforms to ECG recordings in the absence (Figure 2, first row) and presence (Figure 2, second row) of both single- (Figure 2, left column) and multi-echo (Figure 2, right column) BOLD EPI sequences to identify frequencies of confounding MR-related noise. This revealed MR-related noise in frequencies corresponding to the TR and slice acquisition, which was greater in recordings collected during multi-echo sequences than during single-echo sequences (Figure 2D vs. 
2L). The presence and relative impacts of this noise is visually apparent in the difference between recordings collected before and during the two BOLD EPI sequences in Figure 2 (MBSE-ECG in 2A vs. $2 \mathrm{C}$; MBME-ECG in 2I vs. 2K) and evidenced by greater power in the frequencies corresponding with TR and slice acquisition (MBSE-ECG in 2B vs. 2D; MBME-ECG, 2J vs. 2L). These artifacts occur at frequencies equal to (a) the slice frequency which is equal to the number of slices divided by the multiband factor per TR (indicated by circular, blue-green markers), (b) the TR frequency (indicated by triangular, pink markers), and the harmonics of these frequencies. Furthermore, the power of these confounding signals was much greater than in MBSE-ECG recordings and caused greater corruption of the ECG signal (Figure 2C vs $2 \mathrm{~K}$ ).

\section{Denoising electrocardiogram recordings}

We applied BIOPAC-recommended filtering to ECG recordings collected during MBSE and MBME BOLD EPI sequences (MBSE-ECG and MBME-ECG, respectively), via IIR notch filters centered at the slice frequency (Figure 2E-F, M-N). This resulted in an incomplete mitigation of MR-related artifacts, which are still clearly present in the frequency spectra (Figure $2 \mathrm{~F}, \mathrm{~N}$ ).

Based on the identified artifact frequencies, we then adjusted these recommendations to account for the multiband factor of each sequence, with IIR notch filters centered at the frequency corresponding to the number of slices divided by the multiband factor per TR (see equation below) and applied the resultant filter (again, including harmonics) to MBSE-ECG recordings (Figure 2G-H).

$$
v_{\text {slice collection }}=\text { number of slices } \div \text { MB factor } \div \text { TR }
$$

Finally, we applied these adjusted recommendations and additional IIR notch filters centered at the TR frequency to mitigate the effects of additional confounding frequencies present in MBME-ECG recordings, demonstrating the additional need for filtering beyond that which MBSE-ECG requires, in order to obtain MR-denoised ECG recordings simultaneous with MBME BOLD EPI data (Figure 2O-P). 

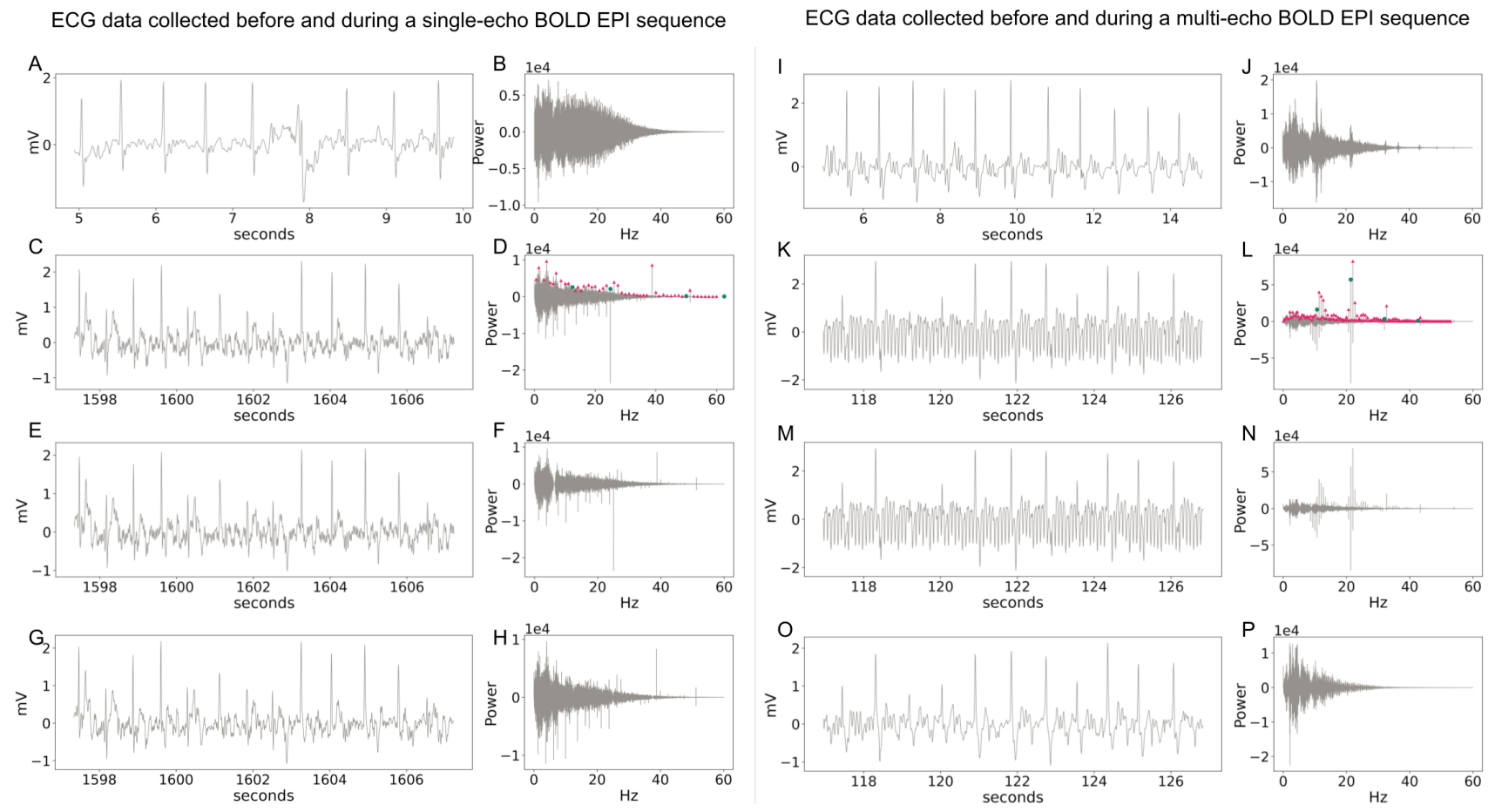

Figure 2. Electrocardiogram recordings through the denoising process. (A) 30 seconds of ECG recordings before the EPI sequence started, after the participant was moved into the scanner bore and (B) the Fourier transform of that recording. (C) 30 seconds of ECG recordings during a single-echo BOLD EPI sequence and (D) the Fourier transform of that recording, demonstrating sequence-related artifacts at the slice frequency (blue-green circles) and TR frequency (pink crosses), and their harmonics. (E) The same 30 seconds of ECG recordings, following application of IIR notch filters to remove the MR-related artifacts, per manufacturer recommendations and (F) the associated power spectrum, which shows remaining MR-related artifacts after filtering. (G) The same 30 seconds, following application of IIR notch filters updated for multiband acquisition and $(\mathrm{H})$ the associated power spectrum, displaying mitigated artifacts, but not complete removal. (I) 30 seconds of ECG recordings from a second individual in the MR environment prior to scanning with a BOLD EPI sequence and $(\mathrm{J})$ the power spectrum of that pre-EPI recording. (K) 30 seconds of ECG recordings from the same individual and scanning session during a multi-echo BOLD EPI sequence data and (L) the power spectrum of that ECG recording, demonstrating MR-related artifacts at the slice frequency (blue-green circles) and TR frequency (pink triangles), and their harmonics. (M) The same 30 seconds of ECG recording, following the application of BIOPAC-recommended filters and $(\mathrm{N})$ the Fourier transform of that recording, displaying the remaining MR-related artifacts. (O) The same 30 seconds of ECG recording, following the application of IIR notch filters at the slice and TR frequencies and $(\mathrm{P})$ the Fourier transform of that cleaned recording, displaying the absence of MR-related artifacts.

\section{Denoising electrodermal activity recordings}

Prior research on EDA recordings collected during single-band, single-echo BOLD sequences has shown minimal MR-related artifacts in EDA data (Robinson et al., 2014). As such, EDA recordings collected simultaneously with fMRI data do not typically require MR-specific denoising. However, a Fourier transform of EDA recordings acquired during the multiband, single-echo BOLD EPI sequence (hereafter MBSE-EDA) in question revealed noise in sequence-specific frequency bands (Figure 3D, L) corresponding to the harmonics of the TR frequency (pink triangles) and, to a lesser extent, of the slice 
collection frequency (blue-green circles). To remove MR-related noise from these EDA recordings, we followed the same filtering procedures as mentioned above (see Denoising electrocardiogram recordings).

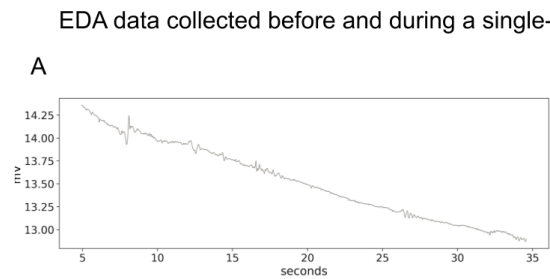

C

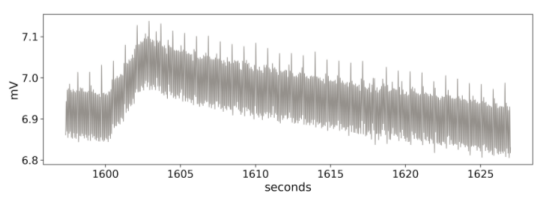

E

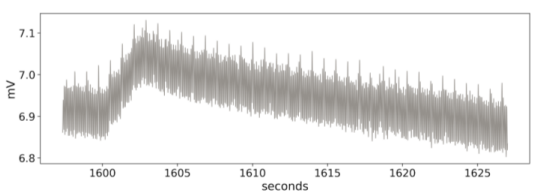

G

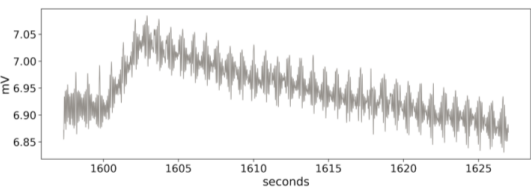

$B$

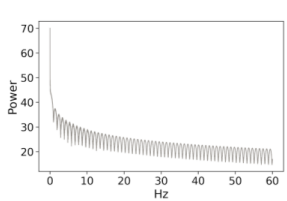

D

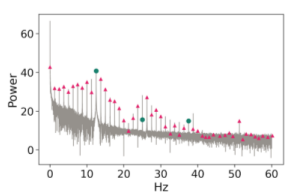

$\mathrm{F}$

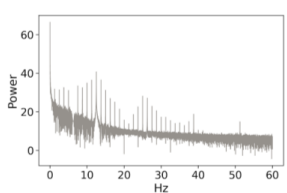

$\mathrm{H}$

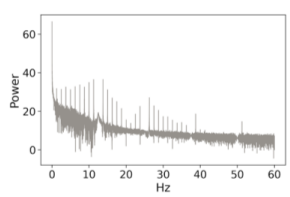

EDA data collected before and during a multi-echo BOLD EPI sequence

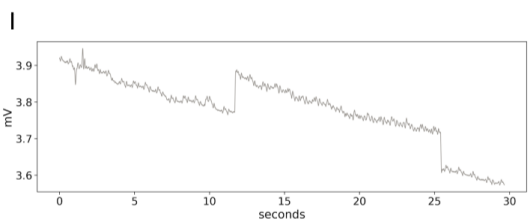

$\mathrm{J}$
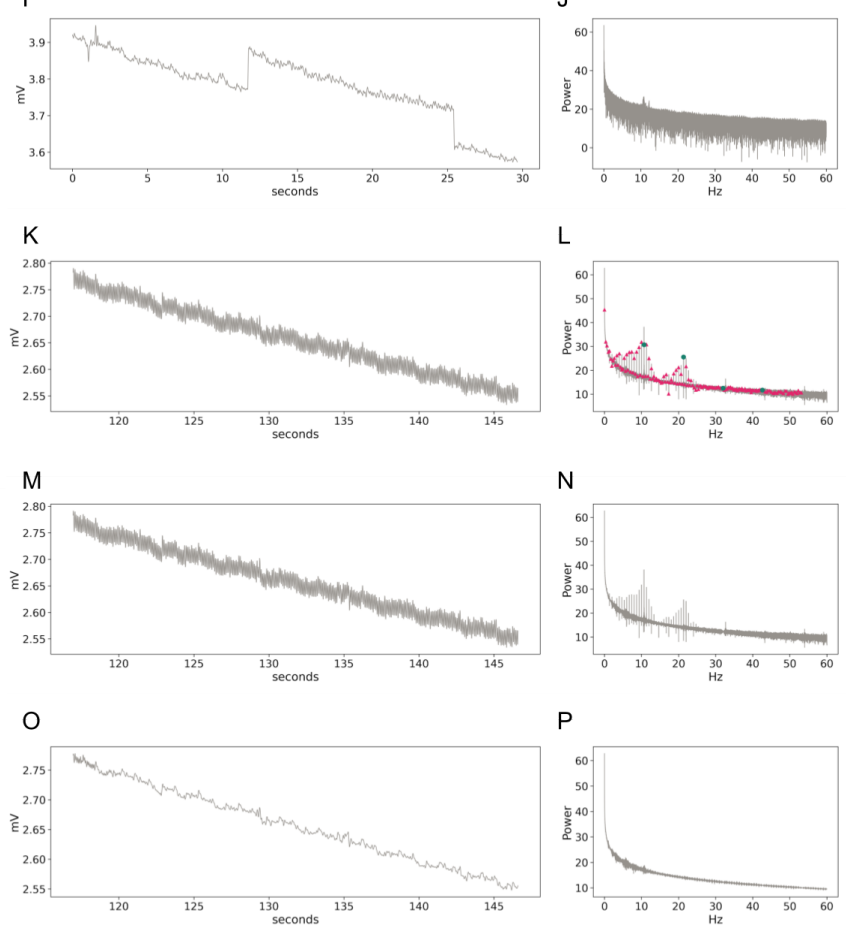

L

N
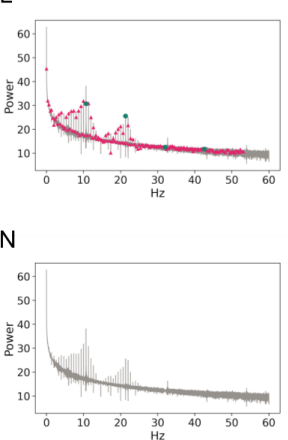

$P$

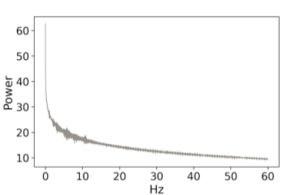

Figure 3. Electrodermal activity recordings through the denoising process. (A) 30 seconds of EDA recordings before the EPI sequence started, after the participant was moved into the scanner bore and (B) the Fourier transform of that recording. (C) 30 seconds of EDA recordings during a single-echo BOLD EPI sequence and (D) the Fourier transform of that recording, demonstrating sequence-related artifacts at the slice frequency (blue-green circles) and TR frequency (pink crosses), and their harmonics. (E) The same 30 seconds of EDA recordings, following application of IIR notch filters to remove the MR-related artifacts, per manufacturer recommendations and (F) the associated power spectrum, which shows remaining MR-related artifacts after filtering. (G) The same 30 seconds of EDA recordings, following the application of IIR notch filters to remove MR-related artifacts, updated to include multiband factor in manufacturer recommendations and $(\mathrm{H})$ the associated power spectrum. (I) 30 seconds of EDA recordings from a second individual in the MR environment prior to scanning with a BOLD EPI sequence and (J) the power spectrum of that pre-EPI recording. (K) 30 seconds of EDA recordings from the same individual and scanning session during a multi-echo BOLD EPI sequence data and (L) the power spectrum of that EDA recording, demonstrating MR-related artifacts at the slice frequency (blue-green circles) and TR frequency (pink crosses), and their harmonics. (M) The same 30 seconds of EDA recording, following the application of BIOPAC-recommended filters and $(\mathrm{N})$ the Fourier transform of that recording, displaying the remaining MR-related artifacts. (O) The same 30 seconds of EDA recording, following the application of IIR notch filters at the slice and TR frequencies and $(\mathrm{P})$ the Fourier transform of that cleaned EDA recording, displaying the absence of MR-related artifacts.

\section{Quantitative assessments of denoised data}

Finally, magnitude squared coherence was computed between each pair of MBSE-ECG recordings across filtering approaches and, separately, between each pair of MBME-ECG recordings. This 
allowed a direct comparison of frequency spectra between (i) filtering approaches, between (ii) filtered recordings and unfiltered recordings, and between (iii) filtered recordings and ECG recordings in the absence of EPI sequences.

Linear dependence of ECG recordings across the denoising approaches described above was assessed using pairwise magnitude squared coherence of the cleaned ECG recordings across filtering approaches, the raw ECG recordings collected during BOLD EPI sequences, and the no-MR recordings (Figure 4). These comparisons between recordings across the denoising process clearly demonstrate the differences in filtering between BIOPAC-recommended and updated approaches for data collected during multiband and multi-echo pulse sequences. MBSE-ECG recordings (Figure 4, lower triangle) retained the same frequency spectra in common with ECG recordings collected in the absence of an MR sequence, regardless of filters applied (BIOPAC-recommended or its multiband equivalent; Figure 4, column 1). Between cleaned MBSE-ECG recordings (Figure 4, upper triangle) and ECG recordings collected in the absence of an MRI sequence (Figure 4, row 1), manufacturer recommendations did not change the frequency spectra in common, when compared with unfiltered MBME-ECG data, but the thoroughly filtered (i.e., with multiband-updated slice frequency filtered and the additional TR frequency filtering) there are more high frequencies in common. Linear dependence between these recordings is lower than between any other pair of recordings. Between raw and cleaned MBSE- and MBME-ECG recordings (Figure 4, second and third columns and rows), there remained more low frequencies $(<250 \mathrm{~Hz})$ in common. However, this may be due to the relatively higher power of lower frequencies in these recordings in general, regardless of filtering approach (see Figure 2). 


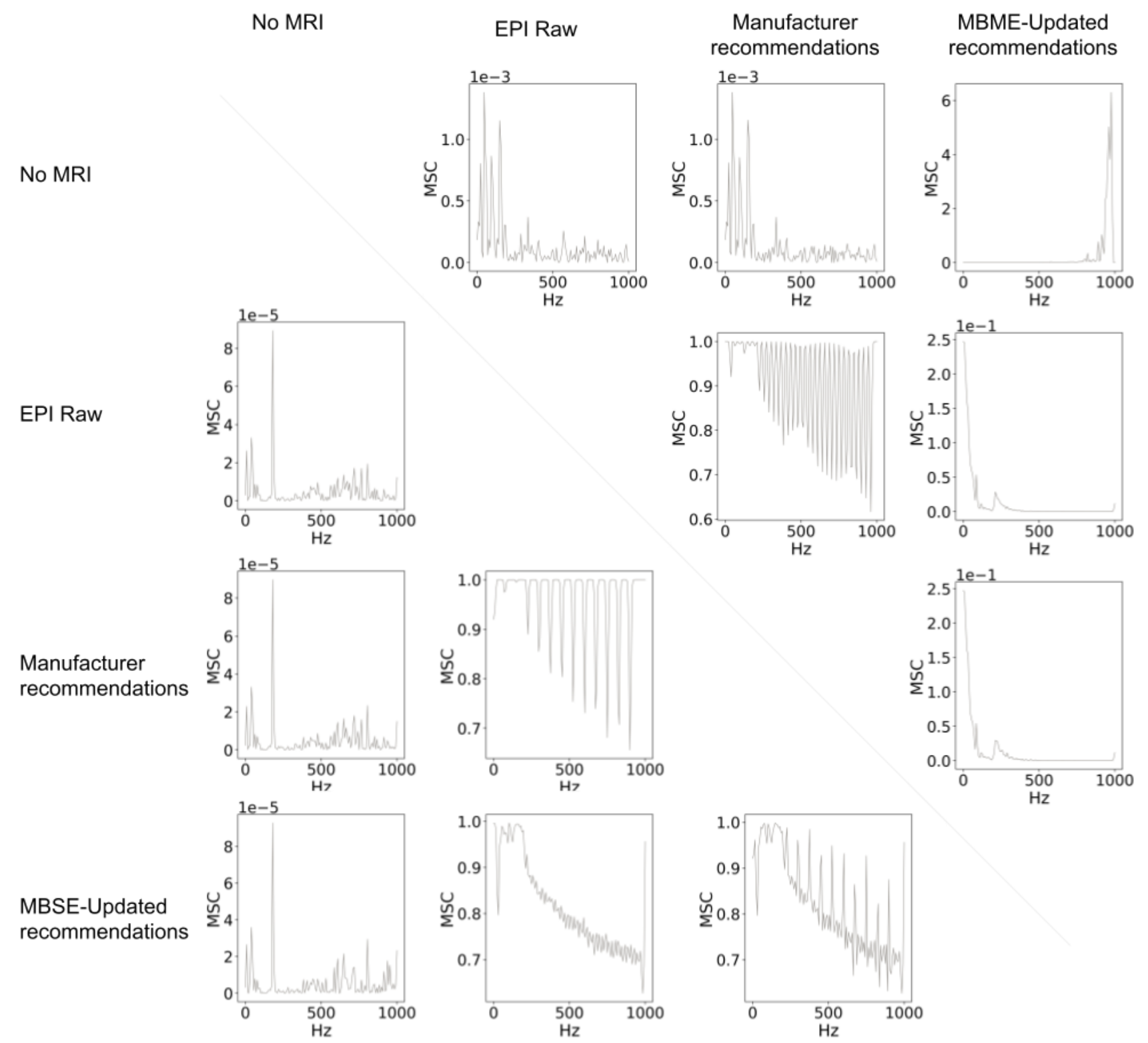

Figure 4. Magnitude squared coherence (MSC) between raw and cleaned ECG recordings. Comparisons between MBSE-ECG recordings are shown below the diagonal; comparisons between MBME-ECG recordings, above the diagonal.

Within the frequency band in which physiologically-relevant signals are found in ECG $(0.5-50 \mathrm{~Hz})$, we assessed the correlations between power spectra across filtering approaches. Average correlation between MBSE-ECG recordings filtered with MB-updated manufacturer recommendations $(r=0.633)$ was slightly higher than that of MBSE-ECG recordings filtered with BIOPAC-recommended filters $(r=$ 0.619), both of which were higher than the average correlation between power spectra of MBSE-ECG and no-MR-ECG recordings $(r=0.573$ ) (Supplemental Table 1, Supplemental Figure 1). Average correlation between power spectra of MBME-ECG recordings filtered with our recommended slice- 
and TR-frequency filters $(r=0.659)$ was higher than the average correlation between no-MR-ECG and MBME-ECG recordings denoised with BIOPAC-recommended filters $(r=0.539)$, both of which were higher than the average correlation between unfiltered MBME-ECG recordings and no-MR- ECG recordings $(r=0.480)$ (Supplemental Table 2, Supplemental Figure 2).

This approach was repeated for each pair of MBSE-EDA recordings across filtering approaches and, separately, between each pair of MBME-EDA recordings.

The magnitude squared coherence of EDA recordings collected before and during BOLD EPI sequences, and across filtering approaches, show linear dependence (i.e., similarity) between pairs of recordings across frequencies. These indicate that, as with MBSE-ECG recordings, MBSE-EDA recordings' similarity to EDA recordings in the absence of an MR sequence do not differ greatly across the cleaning process (Figure 5, column 1). Likewise, MBME-EDA recordings showed greater similarity to EDA recordings in the absence of an MR sequence in higher frequency bands following the sliceand TR-frequency filtering (Figure 5, row 1, MBME-Updated Recommendation). 


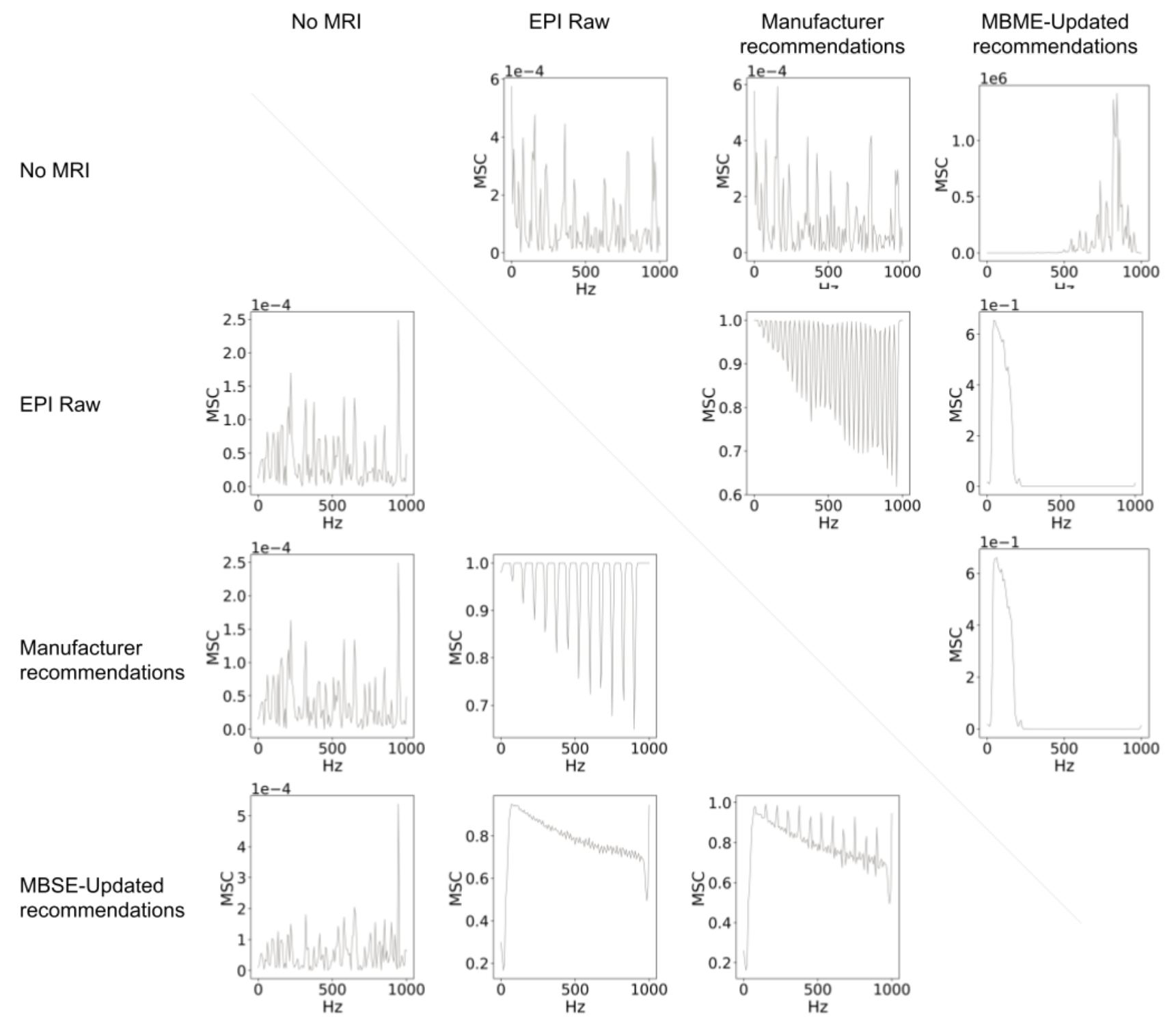

Figure 5. Magnitude squared coherence (MSC) between raw and cleaned EDA recordings. Comparisons between power spectra of MBSE-EDA recordings are shown below the diagonal; comparisons between MBME-EDA recordings, above the diagonal.

Within the frequency band in which physiologically-relevant signals are found in EDA $(<0.5 \mathrm{~Hz})$, we assessed the correlations between power spectra across filtering approaches. Average correlations of MBSE- and MBME-EDA power spectra and no-MR-EDA power spectra were much higher than their -ECG counterparts $\left(r_{M B S E} \geq 0.99, r_{M B M E} \geq 0.999\right)$ with very little variability $\left(\sigma_{r(M B S E)}=0.001, \sigma_{r(M B M E)}=1.84\right.$ $\times 10^{-5}$ ) (Supplemental Tables 4 and 5, Supplemental Figures 4 and 5). Overall, this supports the claim in prior research that the impacts of MR-related artifacts on simultaneously-collected EDA recordings are minimal, although not nonexistent. 


\section{Research Products}

The workflows used to clean these data and create the associated figures are available as a command-line Python script and in interactive Jupyter Notebooks available at:

https://github.com/62442katieb/mbme-physio-denoising/.

These notebooks are additionally available, interactively, at:

https://mybinder.org/v2/gh/62442katieb/mbme-physio-denoising/binder-live.

\section{Discussion}

Here, we assessed the confounding influence of both multiband, single-echo and a multiband, multi-echo BOLD MRI sequences on simultaneously acquired peripheral physiological recordings (e.g., ECG and EDA). These artifacts were demonstrated in recordings collected over the course of several MRI scans, using an MBSE BOLD EPI sequence with a multiband factor of 6 and a MBME BOLD EPI sequence that acquired 4 volumes per RF excitation with a multiband factor of 3 . Two fundamental confounding frequencies were identified, corresponding with the slice frequency and the repetition time of the MRI sequence. Applying a series of notch filters centered at frequencies corresponding to the sequence's TR and slice collection frequency, mimicking a comb band-stop filter (per manufacturer (i.e., BIOPAC) recommendations) provided marked decrease of confounding signals. Based on this, we present an updated set of recommendations for mitigation of pulse sequence-related artifacts in ECG and EDA recordings collected during multiband, multi-echo BOLD MRI scans. These recommendations make it easier for researchers to include physiological recordings during functional MRI studies that capitalize on the improved temporal signal-to-noise ratio (tSNR) of multi-echo pulse sequences and the improvements to temporal resolution made possible by simultaneous multi-slice acquisition. While we did not test these recommendations across a range of pulse sequences with different numbers of echoes and multiband factors, it is likely that our recommendations will generalize across MBME BOLD EPI sequences due to the linear relationship between confounding frequency bands and the sequence's TR and multiband factor.

Building on prior research, we found MRI sequence artifacts in simultaneously collected ECG recordings in frequency bands correspond to the number of asynchronously-collected slices (i.e., the number of slices divided by the multiband factor). Furthermore, these confounding frequencies were of greater power in recordings collected during multi-echo BOLD EPI scans than during single-echo. The impact of these corrupting frequencies can be removed with a series of IIR notch filters corresponding to the TR and slice collection frequencies and their harmonics up to the Nyquist frequency. Further processing is needed in order to distinguish moment-to-moment heart rate and data derived therefrom, but the data have been cleaned of the confounding MR-related artifacts.

Contrary to prior research, we found MRI sequence artifacts in simultaneously collected EDA recordings, both during single- and multi-echo BOLD EPI sequences (Figure 4D, L). These artifacts corresponded with the TR frequency, likely related to the transmission of RF excitation pulses, and the gradient pulses during slice collection. However, the relative power of these confounding frequencies did not differ between MBSE-EDA and MBME-EDA, in contrast to those in the ECG signals (see Figure2). Here, we demonstrate that these artifacts can be removed in the same manner as from 
MBSE- and MBME-ECG recordings with a series of IIR notch filters, mimicking a comb band-stop filter that removes a frequency band and its harmonics up to the Nyquist frequency.

Confounding frequencies corresponding with the TR of the sequence were detected via comparison of the power spectra of ECG and EDA recordings before (Figures 2B and 3B, respectively) and during a MBME BOLD EPI sequence (Figures 2D and 3D, respectively). This frequency is not often mentioned in the simultaneous physiology-fMRI literature as RF pulse-related artifacts are either of a much lesser amplitude than other MR-artifacts or they are filtered out entirely by MRI-specific amplifiers (see Figure 1 for comparison) (Allen et al., 2000; Negishi et al., 2007). However, the RF excitation that precedes slice collection in multiband MRI pulse sequences has a higher amplitude and/or greater total power than that of a single-band sequence. This increased power may explain why the artifact is seen here, in frequency bands corresponding with the sequence TR as shown in Figures $2 \mathrm{~F}$ and 3, but not usually seen in physiological recordings collected simultaneously with single-band BOLD sequences and is not mentioned in prior simultaneous ECG- or EDA-fMRI research or the associated manufacturer recommendations for signal cleaning.

The confounding fundamental frequency identified here corresponds with the slice collection or slice repetition frequency (Allen et al., 2000). This artifact is more commonly seen in ECG recordings collected during fMRI scans, though not in EDA recordings (Robinson et al., 2014). The literature on simultaneous EEG-fMRI acquisition and data cleaning suggests that the magnitude of artifacts due to electromotive force caused by time-varying magnetic field gradients during slice acquisition far surpasses that of the RF excitation pulse (Allen et al., 2000). While this artifact is seen in physiological recordings acquired during single-band BOLD sequences, as well, the power of the harmonics of this confounding frequency are much greater in data collected during multiband BOLD sequences. Although slice collection in multi-echo GRE-EPI sequences is more prolonged over the course of a timepoint of data acquisition, due to the acquisition of multiple volumes of data per RF excitation pulse, the duration of slice collection is short $(<75 \mathrm{~ms})$ compared to the repetition time (1500ms). As such, the confounding frequency associated with time-varying gradients is centered on the slice frequency ( slices / MB factor / TR) and confounding frequencies associated with individual echoes were not observed. Notch filters centered at the slice frequency and its harmonics sufficiently removed the artifact caused by shifting gradient fields.

\section{Limitations and Considerations}

The temporal resolutions of each electrophysiological $(1000-5000 \mathrm{~Hz})$ and fMRI $(0.5-1.5 \mathrm{~Hz})$ data complicate psychophysiological analyses. First, in relating physiological processes to BOLD signal fluctuations, accounting for differences in the timing of individual slice collection, typically performed in the beginning of fMRI preprocessing (Jones et al., 2008; Parker et al., 2017; Sladky et al., 2011), becomes crucial. Second, physiological data should be downsampled for such investigations. fMRI data are collected with a TR between $500 \mathrm{~ms}$ and $3 \mathrm{~s}$ and while multiband acquisition can shorten TRs, multi-echo acquisition often lengthens TRs. When TRs exceed 2 seconds, the temporal resolution of fMRI data becomes low enough to induce aliasing in biologically-relevant frequency bands of physiological data downsampled to match. Researchers should proceed with caution when this is the case. 
On another note, researchers collecting data regarding heart rate and cardiac pulsations can avoid MR artifacts entirely by using a photoplethysmograph, which collects optical instead of electrical measurements.

\section{Conclusions}

While MBSE and MBME pulse sequences introduce more complicated artifacts into simultaneously acquired electrophysiological recordings than can be addressed with current manufacturer recommendations, the data presented here suggest that these artifacts are predictable and their effects can be greatly mitigated with notch filters centered at their fundamental frequencies and harmonics. By targeting the slice acquisition frequency, updated to account for multiband factor, and, especially in the case of MBME-simultaneous recordings, TR frequency, researchers should be able to remove significant MR-related artifacts from ECG and EDA data collected during fMRI scans. Recommendations such as those demonstrated here allow researchers to capitalize on the improved SNR afforded by MBSE and MBME BOLD sequences, while including rich information concerning a participant's peripheral, visceral state. 
16

\section{Declaration of Conflicting Interests}

The author(s) declared no potential conflicts of interest with respect to the research, authorship, and/or publication of this article.

\section{Funding}

Funding for this project was provided by the Florida International University Center for Imaging Science and FIU's Office of Research and Economic Development; we also acknowledge federal support from NIH U01 DA041156, NIH R01 DA041353, NSF 1631325, and NSF CNS 1532061. Thanks to the FIU Instructional \& Research Computing Center (IRCC, http:/ /ircc.fiu.edu) for providing the HPC and computing resources that contributed to the research results reported within this paper.

\section{ORCID iD}

Katherine L. Bottenhorn (iD https://orcid.org/0000-0003-1024-4562

\section{Supplemental Material}

Supplemental material for this article is available online. 


\section{References}

Abreu, R., Nunes, S., Leal, A., \& Figueiredo, P. (2017). Physiological noise correction using ECG-derived respiratory signals for enhanced mapping of spontaneous neuronal activity with simultaneous EEG-fMRI. NeuroImage, 154, 115-127.

https:/ / doi.org/10.1016/j.neuroimage.2016.08.008

Allen, P. J., Josephs, O., \& Turner, R. (2000). A method for removing imaging artifact from continuous EEG recorded during functional MRI. NeuroImage, 12(2), 230-239. https:/ / doi.org/10.1006/nimg.2000.0599

Auerbach, E. J., Xu, J., Yacoub, E., Moeller, S., \& Uğurbil, K. (2013). Multiband accelerated spin-echo echo planar imaging with reduced peak RF power using time-shifted RF pulses. Magnetic Resonance in Medicine, 69(5), 1261-1267. https:/ /doi.org/10.1002/mrm.24719

Blair, K. S., Geraci, M., Smith, B. W., Hollon, N., DeVido, J., Otero, M., Blair, J. R., \& Pine, D. S. (2012). Reduced Dorsal Anterior Cingulate Cortical Activity During Emotional Regulation and Top-Down Attentional Control in Generalized Social Phobia, Generalized Anxiety Disorder, and Comorbid Generalized Social Phobia/Generalized Anxiety Disorder. Biological Psychiatry, 72(6), 476-482. https://doi.org/10.1016/j.biopsych.2012.04.013

Boyacioğlu, R., Schulz, J., Koopmans, P. J., Barth, M., \& Norris, D. G. (2015). Improved sensitivity and specificity for resting state and task fMRI with multiband multi-echo EPI compared to multi-echo EPI at 7 T. NeuroImage, 119, 352-361.

https://doi.org/10.1016/j.neuroimage.2015.06.089

Casey, B. J., Cannonier, T., Conley, M. I., Cohen, A. O., Barch, D. M., Heitzeg, M. M., Soules, M. E., Teslovich, T., Dellarco, D., Garavan, H., Orr, C. A., Wager, T. D., Banich, M. T., Speer, N. K., Sutherland, M. T., Riedel, M. C., Dick, A. S., Bjork, J. M., Thomas, K. M., ... Dale, A. M. 
(2018). The Adolescent Brain Cognitive Development (ABCD) study: Imaging acquisition across 21 sites. Developmental Cognitive Neuroscience, 32, 43-54. https://doi.org/10.1016/J.DCN.2018.03.001

Cohen, A. D., Nencka, A. S., Lebel, R. M., \& Wang, Y. (2017). Multiband multi-echo imaging of simultaneous oxygenation and flow timeseries for resting state connectivity. PLOS ONE, 12(3), e0169253. https:/ / doi.org/10.1371/journal.pone.0169253

Desmond, J. E., \& Glover, G. H. (2002). Estimating sample size in functional MRI (fMRI) neuroimaging studies: Statistical power analyses. Journal of Neuroscience Methods, 118(2), 115-128. https:/ / doi.org/10.1016/s0165-0270(02)00121-8

Duffer, M., \& Duffer, R. (2017). Stranger things. In Nature Materials. Netflix.

Frank, M. J., Seeberger, L. C., \& O’Reilly, R. C. (2004). By Carrot or by Stick: Cognitive Reinforcement Learning in Parkinsonism. Science, 306(5703), 1940-1943. https:/ / doi.org/10.1126/science.1102941

Goldstein, J. M., Jerram, M., Poldrack, R., Ahern, T., Kennedy, D. N., Seidman, L. J., \& Makris, N. (2005). Hormonal cycle modulates arousal circuitry in women using functional magnetic resonance imaging. The Journal of Neuroscience: The Official Journal of the Society for Neuroscience, 25(40), 9309-9316. https: / / doi.org/10.1523/JNEUROSCI.2239-05.2005

Harris, C. R., Millman, K. J., van der Walt, S. J., Gommers, R., Virtanen, P., Cournapeau, D., Wieser, E., Taylor, J., Berg, S., Smith, N. J., Kern, R., Picus, M., Hoyer, S., van Kerkwijk, M. H., Brett, M., Haldane, A., del Río, J. F., Wiebe, M., Peterson, P., ... Oliphant, T. E. (2020). Array programming with NumPy. Nature, 585(7825), 357-362. https:/ / doi.org/10.1038/s41586-020-2649-2

Hu, C., Tokoglu, F., Scheinost, D., Qiu, M., Shen, X., Peters, D. C., Galiana, G., \& Constable, R. T. 
(2019). Dynamic-flip-angle ECG-gating with nuisance signal regression improves resting-state BOLD functional connectivity mapping by reducing cardiogenic noise. Magnetic Resonance in Medicine, 82(3), 911-923. https:/ /doi.org/10.1002/mrm.27775

Jones, T. B., Bandettini, P. A., \& Birn, R. M. (2008). Integration of motion correction and physiological noise regression in fMRI. NeuroImage, 42(2), 582-590. https:/ / doi.org/10.1016/j.neuroimage.2008.05.019

Kraus, T., Hösl, K., Kiess, O., Schanze, A., Kornhuber, J., \& Forster, C. (2007). BOLD fMRI deactivation of limbic and temporal brain structures and mood enhancing effect by transcutaneous vagus nerve stimulation. Journal of Neural Transmission (Vienna, Austria: 1996), 114(11), 1485-1493. https:/ / doi.org/10.1007/s00702-007-0755-z

Kundu, P., Voon, V., Balchandani, P., Lombardo, M. V., Poser, B. A., \& Bandettini, P. A. (2017). Multi-echo fMRI: A review of applications in fMRI denoising and analysis of BOLD signals. NeuroImage, 154, 59-80. https://doi.org/10.1016/j.neuroimage.2017.03.033

Kuoppa, P., Niskanen, E., Karjalainen, P., \& Tarvainen, M. P. (2012). Cerebral cortex and sub-cortex lateralization in cardiovascular regulation: Correlations of BOLD fMRI and heart rate variability. Conference Proceedings: ... Annual International Conference of the IEEE Engineering in Medicine and Biology Society. IEEE Engineering in Medicine and Biology Society. Annual Conference, 2012, 3412-3415. https://doi.org/10.1109/EMBC.2012.6346698

Lane, R. D., Weidenbacher, H., Smith, R., Fort, C., Thayer, J. F., \& Allen, J. J. B. (2013). Subgenual anterior cingulate cortex activity covariation with cardiac vagal control is altered in depression. Journal of Affective Disorders, 150(2), 565-570. https:/ / doi.org/10.1016/j.jad.2013.02.005

Mobascher, A., Brinkmeyer, J., Warbrick, T., Musso, F., Schlemper, V., Wittsack, H. J., Saleh, A., 
Schnitzler, A., \& Winterer, G. (2010). Brain activation patterns underlying fast habituation to painful laser stimuli. International Journal of Psychophysiology: Official Journal of the International Organization of Psychophysiology, 75(1), 16-24.

https:/ / doi.org/10.1016/j.ijpsycho.2009.10.008

Mobascher, A., Brinkmeyer, J., Warbrick, T., Musso, F., Wittsack, H. J., Stoermer, R., Saleh, A., Schnitzler, A., \& Winterer, G. (2009). Fluctuations in electrodermal activity reveal variations in single trial brain responses to painful laser stimuli-A fMRI/EEG study. NeuroImage, 44(3), 1081-1092. https:/ / doi.org/10.1016/j.neuroimage.2008.09.004

Mulcahy, J. S., Larsson, D. E. O., Garfinkel, S. N., \& Critchley, H. D. (2019). Heart rate variability as a biomarker in health and affective disorders: A perspective on neuroimaging studies. NeuroImage, 202, 116072. https:/ /doi.org/10.1016/j.neuroimage.2019.116072

Napadow, V., Dhond, R., Conti, G., Makris, N., Brown, E. N., \& Barbieri, R. (2008). Brain correlates of autonomic modulation: Combining heart rate variability with fMRI. NeuroImage, 42(1), 169-177. https:/ / doi.org/10.1016/j.neuroimage.2008.04.238

NBCLab/eirt-task. (2020). [XSLT]. Neuroinformatics and Brain Connectivity Lab.

https:/ /github.com/NBCLab/eirt-task (Original work published 2020)

NBCLab/film-viewing-task. (2020). [Python]. Neuroinformatics and Brain Connectivity Lab.

https:/ /github.com/NBCLab/film-viewing-task (Original work published 2019)

Negishi, M., Pinus, B. I., Pinus, A. B., \& Constable, R. T. (2007). Origin of the Radio Frequency Pulse Artifact in Simultaneous EEG-fMRI Recording: Rectification at the Carbon-Metal Interface. IEEE Transactions on Bio-Medical Engineering, 54(9), 1725-1727. https://doi.org/10.1109/TBME.2007.891940

Ochsner, K. N., Bunge, S. A., Gross, J. J., \& Gabrieli, J. D. E. (2002). Rethinking Feelings: An fMRI 
Study of the Cognitive Regulation of Emotion. Journal of Cognitive Neuroscience, 14(8),

1215-1229. https://doi.org/10.1162/089892902760807212

Ochsner, K. N., Ray, R. D., Cooper, J. C., Robertson, E. R., Chopra, S., Gabrieli, J. D. E., \& Gross, J. J. (2004). For better or for worse: Neural systems supporting the cognitive down- and up-regulation of negative emotion. NeuroImage, 23(2), 483-499.

https:/ / doi.org/10.1016/j.neuroimage.2004.06.030

Olafsson, V., Kundu, P., Wong, E. C., Bandettini, P. A., \& Liu, T. T. (2015). Enhanced Identification of BOLD-like Components with Multi-Echo Simultaneous Multi-Slice (MESMS) fMRI and Multi-Echo ICA. NeuroImage, 112, 43-51. https:/ / doi.org/10.1016/j.neuroimage.2015.02.052

Parker, D., Liu, X., \& Razlighi, Q. R. (2017). Optimal slice timing correction and its interaction with fMRI parameters and artifacts. Medical Image Analysis, 35, 434-445.

https: / / doi.org/10.1016/j.media.2016.08.006

Perlaki, G., Orsi, G., Schwarcz, A., Bodi, P., Plozer, E., Biczo, K., Aradi, M., Doczi, T., Komoly, S., Hejjel, L., Kovacs, N., \& Janszky, J. (2015). Pain-related autonomic response is modulated by the medial prefrontal cortex: An ECG-fMRI study in men. Journal of the Neurological Sciences, 349(1-2), 202-208. https:/ / doi.org/10.1016/j.jns.2015.01.019

Robinson, J. L., Miller, M. W., Beyers, R., Grand, K., Kirby, L. A., Macy, A., \& Graap, K. (2014). NEUROPSYCHOPHYSIOLOGICAL MAPPING: CONCOMMITANT PSYCHOPHYSIOLOGICAL RECORDING AND SUBMILLIMETER FUNCTIONAL MAGNETIC RESONANCE IMAGING (FMRI) AT 7T. 51, S78-S78.

Sclocco, R., Garcia, R. G., Kettner, N. W., Isenburg, K., Fisher, H. P., Hubbard, C. S., Ay, I., Polimeni, J. R., Goldstein, J., Makris, N., Toschi, N., Barbieri, R., \& Napadow, V. (2019). The influence of respiration on brainstem and cardiovagal response to auricular vagus nerve stimulation: 
A multimodal ultrahigh-field (7T) fMRI study. Brain Stimulation, 12(4), 911-921.

https: / / doi.org/10.1016/j.brs.2019.02.003

Shokri-Kojori, E., Tomasi, D., \& Volkow, N. D. (2018). An Autonomic Network: Synchrony Between Slow Rhythms of Pulse and Brain Resting State Is Associated with Personality and Emotions. Cerebral Cortex (New York, NY), 28(9), 3356-3371.

https: / /doi.org/10.1093/cercor/bhy144

Sladky, R., Friston, K. J., Tröstl, J., Cunnington, R., Moser, E., \& Windischberger, C. (2011).

Slice-timing effects and their correction in functional MRI. NeuroImage, 58(2), 588-594. https:/ / doi.org/10.1016/j.neuroimage.2011.06.078

Valenza, G., Nardelli, M., Lanatà, A., Gentili, C., Bertschy, G., Paradiso, R., \& Scilingo, E. P. (2014). Wearable Monitoring for Mood Recognition in Bipolar Disorder Based on History-Dependent Long-Term Heart Rate Variability Analysis. IEEE Journal of Biomedical and Health Informatics, 18(5), 1625-1635. https:/ / doi.org/10.1109/JBHI.2013.2290382

Virtanen, P., Gommers, R., Oliphant, T. E., Haberland, M., Reddy, T., Cournapeau, D., Burovski, E., Peterson, P., Weckesser, W., Bright, J., van der Walt, S. J., Brett, M., Wilson, J., Millman, K. J., Mayorov, N., Nelson, A. R. J., Jones, E., Kern, R., Larson, E., ... van Mulbregt, P. (2020). SciPy 1.0: Fundamental algorithms for scientific computing in Python. Nature Methods, 17(3), 261-272. https:/ /doi.org/10.1038/s41592-019-0686-2

Wong, S. W. H., Xue, G., \& Bechara, A. (2011). Integrating fMRI with psychophysiological measurements in the study of decision making. Journal of Neuroscience, Psychology, and Economics, 4(2), 85-94. https:/ / doi.org/10.1037/a0023525 